\title{
SECTORIALNESS OF SECOND ORDER ELLIPTIC OPERATORS IN DIVERGENCE FORM
}

\author{
NOBORU OKAZAWA
}

(Communicated by Palle E. T. Jorgensen)

\begin{abstract}
A sectorial estimate is given to second order linear elliptic differential operators of divergence form. The estimate is a slight improvement of Pazy's. The obtained constant depends on $p$ of the space $L^{p}(\Omega) \quad(1<p<\infty)$ and does not depend on the operators themselves. The same constant has appeared in the sectorial estimate for second order linear ordinary differential operators due to Fattorini.

The result is in connection with Stein's estimate of the analytic semigroups generated by linear elliptic differential operators.
\end{abstract}

\section{INTRODUCTION}

Let $\Omega$ be a bounded domain in $R^{m}$ with smooth boundary, and let $A(x, D)$ be the second order differential operator of divergence form

$$
A(x, D) u=-\sum_{j, k=1}^{m} \frac{\partial}{\partial x_{k}}\left[a_{j k}(x) \frac{\partial u}{\partial x_{j}}\right] .
$$

We assume that the coefficients $a_{j k}(x)=a_{k j}(x)$ are real-valued and continuously differentiable on $\bar{\Omega}$, and that $A(x, D)$ is uniformly elliptic or degenerate elliptic, i.e., there is a constant $C_{0} \geqq 0$ such that for all $\xi \in R^{m}$,

$$
\sum_{j, k=1}^{m} a_{j k}(x) \xi_{j} \xi_{k} \geqq C_{0}|\xi|^{2}:=C_{0} \sum_{j=1}^{m} \xi_{j}^{2} .
$$

Let $1<p<\infty$ and consider the linear operator in $L^{p}(\Omega)$ :

$$
\begin{gathered}
D\left(A_{p}\right)=W^{2, p}(\Omega) \cap W_{0}^{1, p}(\Omega), \\
\left(A_{p} u\right)(x)=A(x, D) u(x) \quad \text { for } u \in D\left(A_{p}\right),
\end{gathered}
$$

Received by the editors April 14, 1990 and, in revised form, September 20, 1990.

1980 Mathematics Subject Classification (1985 Revision). Primary 35J25, 47B45; Secondary 47D05.

Key words and phrases. Second order elliptic operators, uniform and degenerate ellipticity, sectorial operators, analytic contraction semigroups. 
where $W^{k, p}(\Omega)$ is the usual Sobolev space and $W_{0}^{k, p}(\Omega)$ is the completion of $C_{0}^{\infty}(\Omega)$ with respect to the norm of $W^{k, p}(\Omega) \quad(k=1,2)$.

In particular, if $C_{0}>0$, then in his book [7] Pazy proved the following estimates: for $u \in D\left(A_{p}\right), \operatorname{Re}\left(A_{p} u,|u|^{p-2} u\right) \geqq 0$ and

$$
\left|\operatorname{Im}\left(A_{p} u,|u|^{p-2} u\right)\right| \leqq \frac{M|p-2|}{2 C_{0} \sqrt{p-1}} \operatorname{Re}\left(A_{p} u,|u|^{p-2} u\right),
$$

where $M=\max \left\{\left|a_{j k}(x)\right| ; x \in \bar{\Omega}, 1 \leqq j, k \leqq m\right\}$ (see [7, Proof of Theorem 7.3.6]).

The purpose of this note is to show that (4) holds independently of the constants $C_{0}$ and $M$ :

$$
\left|\operatorname{Im}\left(A_{p} u,|u|^{p-2} u\right)\right| \leqq \frac{|p-2|}{2 \sqrt{p-1}} \operatorname{Re}\left(A_{p} u,|u|^{p-2} u\right)
$$

for $u \in D\left(A_{p}\right)$. So we see that (5) holds even in the degenerate elliptic case. Then it follows that the "generalized" numerical range of $A_{p}$ is contained in the sector

$$
|\arg \zeta| \leqq \omega_{p}:=\tan ^{-1}(|p-2| / 2 \sqrt{p-1}),
$$

i.e., $A_{p}$ is sectorial of type $S\left(\tan \omega_{p}\right)$ in the sense of Goldstein [2, Definition 1.5.8] (see also Kato [5, V-§3.10]).

Let $C_{0}>0$. Then we show further that when $p \geqq 2$,

$$
\begin{aligned}
\operatorname{Re}\left(A_{p} u,|u|^{p-2} u\right) \geqq & C_{0} \operatorname{Re}\left(-\Delta u,|u|^{p-2} u\right) \\
= & C_{0} \int_{\Omega}|u(x)|^{p-2}|\operatorname{grad} u(x)|^{2} d x \\
& +(p-2) C_{0} \int_{\Omega}|u(x)|^{p-2}|\operatorname{grad}| u(x)||^{2} d x,
\end{aligned}
$$

and when $1<p<2$,

$$
\begin{aligned}
& \operatorname{Re}\left(A_{p} u,|u|^{p-2} u\right) \\
& \quad \geqq(p-1) C_{0} \lim _{\delta \downarrow 0} \int_{\Omega}\left(|u(x)|^{2}+\delta\right)^{(p-2) / 2}|\operatorname{grad} u(x)|^{2} d x .
\end{aligned}
$$

Here $\Delta$ is the Laplacian; note that $A(x, D)=-\Delta$ if $a_{j k}(x)=\delta_{j k}$ (the Kronecker delta). Since $A_{p}$ is $m$-accretive (see Pazy [7, Theorem 7.3.6]), $A_{p}$ is $m$-sectorial in the sense of $[2,5]$. Therefore, $-A_{p}$ generates an analytic (or a holomorphic) contraction semigroup $\left\{T_{p}(t)\right\}$ on $L^{p}(\Omega)$, i.e., $T_{p}(t)$ is analytic in the sector $|\arg t|<(\pi / 2)-\omega_{p}$ and

$$
\left\|T_{p}(t)\right\|_{p} \leqq 1 \text { for }|\arg t| \leqq(\pi / 2)-\omega_{p}
$$

(see [2, Theorem 1.5.9; 5 , Theorem IX-1.24]). Twenty years ago Stein noticed in [8, III- $\S 2$, Theorem 1, and the proof on p. 71] that

$$
\left\|T_{p}(t)\right\|_{p} \leqq 1 \quad \text { for }|\arg t| \leqq \frac{\pi}{2}\left(1-\left|\frac{2}{p}-1\right|\right)
$$


as a consequence of his interpolation theorem. After a simple computation we see that

$$
\tan ^{-1}\left(\frac{|p-2|}{2 \sqrt{p-1}}\right) \leqq \frac{\pi}{2}\left|\frac{2}{p}-1\right| .
$$

Namely, (8) is an improvement of (9).

\section{Sectorial estimates}

Let $A_{p}$ be defined by (2) and (3). Then for $u \in D\left(A_{p}\right)$,

$$
\begin{aligned}
\left(A_{p} u,|u|^{p-2} u\right) & =-\int_{\Omega}|u(x)|^{p-2} \overline{u(x)} \sum_{j, k=1}^{m} \frac{\partial}{\partial x_{k}}\left[a_{j k}(x) \frac{\partial u}{\partial x_{j}}\right] d x \\
& =\lim _{\delta \downarrow 0} I_{p}(u, \delta),
\end{aligned}
$$

where

$$
I_{p}(u, \delta)=-\int_{\Omega}\left(|u(x)|^{2}+\delta\right)^{\frac{p-2}{2}} \overline{u(x)} \sum_{j, k=1}^{m} \frac{\partial}{\partial x_{k}}\left[a_{j k}(x) \frac{\partial u}{\partial x_{j}}\right] d x .
$$

Here we have to take $\delta>0$ when $1<p<2$, and $\delta=0$ when $p \geqq 2$. Since $u \in W_{0}^{1, p}(\Omega)$, we have

$$
\begin{aligned}
I_{p}(u, \delta)=\int_{\Omega} & \left(|u(x)|^{2}+\delta\right)^{\frac{p-2}{2}} \sum_{j, k=1}^{m} a_{j k}(x) \frac{\partial u}{\partial x_{j}} \frac{\overline{\partial u}}{\partial x_{k}} d x \\
& +(p-2) \int_{\Omega}\left(|u(x)|^{2}+\delta\right)^{\frac{p-4}{2}}|u(x)| \overline{u(x)} \sum_{j, k=1}^{m} a_{j k}(x) \frac{\partial u}{\partial x_{j}} \frac{\partial|u|}{\partial x_{k}} d x ;
\end{aligned}
$$

note that $|u| \in W_{0}^{1, p}(\Omega)$ (Stampacchia's Lemma; see Gilbarg-Trudinger [3, Lemma 7.6]). The first term on the right-hand side of (10) is real. In fact, it is equal to

$$
\begin{aligned}
\int_{\Omega}\left(|u(x)|^{2}+\delta\right)^{\frac{p-2}{2}} \sum_{j, k=1}^{m} a_{j k}(x)[ & \left(\operatorname{Re} \frac{\partial u}{\partial x_{j}}\right)\left(\operatorname{Re} \frac{\partial u}{\partial x_{k}}\right) \\
& \left.+\left(\operatorname{Im} \frac{\partial u}{\partial x_{j}}\right)\left(\operatorname{Im} \frac{\partial u}{\partial x_{k}}\right)\right] d x,
\end{aligned}
$$

and by the ellipticity (1), it is larger than

$$
C_{0} \int_{\Omega}\left(|u(x)|^{2}+\delta\right)^{\frac{p-2}{2}}|\operatorname{grad} u(x)|^{2} d x .
$$

Setting

$$
H_{p}(u, \delta)=\int_{\Omega}\left(|u(x)|^{2}+\delta\right)^{\frac{p-4}{2}}|u(x)| \overline{u(x)} \sum_{j, k=1}^{m} a_{j k}(x) \frac{\partial u}{\partial x_{j}} \frac{\partial|u|}{\partial x_{k}} d x
$$


and noting that $2 \operatorname{Re} \overline{u(x)} \frac{\partial u}{\partial x_{j}}=\frac{\partial}{\partial x_{j}}|u(x)|^{2}$, we have

$$
\operatorname{Re} H_{p}(u, \delta)=\int_{\Omega}\left(|u(x)|^{2}+\delta\right)^{\frac{p-4}{2}}|u(x)|^{2} \sum_{j, k=1}^{m} a_{j k}(x) \frac{\partial|u|}{\partial x_{j}} \frac{\partial|u|}{\partial x_{k}} d x
$$

It follows from $(10)$ that

$$
\begin{gathered}
\operatorname{Re} I_{p}(u, \delta)-(p-2) \operatorname{Re} H_{p}(u, \delta) \\
=\int_{\Omega}\left(|u(x)|^{2}+\delta\right)^{\frac{p-2}{2}} \sum_{j, k=1}^{m} a_{j k}(x) \frac{\partial u}{\partial x_{j}} \frac{\overline{\partial u}}{\partial x_{k}} d x, \\
\operatorname{Im} I_{p}(u, \delta)=(p-2) \operatorname{Im} H_{p}(u, \delta) .
\end{gathered}
$$

Here we can prove that

$$
\operatorname{Re} H_{p}(u, \delta) \leqq \int_{\Omega}\left(|u(x)|^{2}+\delta\right)^{\frac{p-4}{2}}|u(x)|^{2} \sum_{j, k=1}^{m} a_{j k}(x) \frac{\partial u}{\partial x_{j}} \frac{\overline{\partial u}}{\partial x_{k}} d x
$$

In fact, we have

$$
\begin{aligned}
\left|\operatorname{Re} H_{p}(u, \delta)\right|^{2} & \leqq\left|H_{p}(u, \delta)\right|^{2} \\
& \leqq\left[\int_{\Omega}\left(|u(x)|^{2}+\delta\right)^{\frac{p-4}{2}}|u(x)|^{2} \times\left|\sum_{j, k=1}^{m} a_{j k}(x) \frac{\partial u}{\partial x_{j}} \frac{\partial|u|}{\partial x_{k}}\right| d x\right]^{2} .
\end{aligned}
$$

Since $\left(a_{j k}(x)\right)$ is a nonnegative definite symmetric matrix, we can apply the Schwarz inequality:

$$
\begin{aligned}
& \left|\sum_{j, k=1}^{m} a_{j k}(x) \frac{\partial u}{\partial x_{j}} \frac{\partial|u|}{\partial x_{k}}\right|^{2} \\
& \quad \leqq\left(\sum_{j, k=1}^{m} a_{j k}(x) \frac{\partial u}{\partial x_{j}} \frac{\overline{\partial u}}{\partial x_{k}}\right)\left(\sum_{j, k=1}^{m} a_{j k}(x) \frac{\partial|u|}{\partial x_{j}} \frac{\partial|u|}{\partial x_{k}}\right) .
\end{aligned}
$$

Therefore it follows from (11) that

$$
\begin{aligned}
\left|\operatorname{Re} H_{p}(u, \delta)\right|^{2} & \leqq\left|H_{p}(u, \delta)\right|^{2} \\
& \leqq\left[\int_{\Omega}\left(|u(x)|^{2}+\delta\right)^{\frac{p-4}{2}}|u(x)|^{2} \sum_{j, k=1}^{m} a_{j k}(x)\left|\frac{\partial u}{\partial x_{j}} \frac{\overline{\partial u}}{\partial x_{k}}\right| d x\right] \operatorname{Re} H_{p}(u, \delta) .
\end{aligned}
$$

Hence we obtain (14). Now let $p \geqq 2$. Then we see from (11), (12), and (1) that

$$
\begin{aligned}
\operatorname{Re} I_{p}(u, \delta) \geqq & C_{0} \int_{\Omega}|u(x)|^{p-2}|\operatorname{grad} u(x)|^{2} d x \\
& +\left.(p-2) C_{0} \int_{\Omega}|u(x)|^{p-2}|\operatorname{grad}| u(x)\right|^{2} d x, \\
= & C_{0} \operatorname{Re}\left(-\Delta u,|u|^{p-2} u\right)
\end{aligned}
$$


Next let $1<p<2$. Then, since $p-2<0$, it follows from (12), (14), and (1) that

$$
\begin{aligned}
\operatorname{Re} I_{p}(u, \delta) & \geqq(p-1) \int_{\Omega}\left(|u(x)|^{2}+\delta\right)^{\frac{p-2}{2}} \sum_{j, k=1}^{m} a_{j k}(x) \frac{\partial u}{\partial x_{j}} \frac{\overline{\partial u}}{\partial x_{k}} d x \\
& \geqq(p-1) C_{0} \int_{\Omega}\left(|u(x)|^{2}+\delta\right)^{\frac{p-2}{2}}|\operatorname{grad} u(x)|^{2} d x
\end{aligned}
$$

Thus we can obtain (6) and (7).

Now we estimate the $\operatorname{Im} I_{p}(u, \delta)$. If follows from (12), (13), and (15) that

$$
\begin{aligned}
&(p-2)^{-2}\left|\operatorname{Im} I_{p}(u, \delta)\right|^{2}=\left|H_{p}(u, \delta)\right|^{2}-\left|\operatorname{Re} H_{p}(u, \delta)\right|^{2} \\
& \leqq\left[\operatorname{Re} I_{p}(u, \delta)-(p-2) \operatorname{Re} H_{p}(u, \delta)\right] \operatorname{Re} H_{p}(u, \delta)-\left|\operatorname{Re} H_{p}(u, \delta)\right|^{2} \\
& \leqq\left|\operatorname{Re} H_{p}(u, \delta)\right| \cdot\left|\operatorname{Re} I_{p}(u, \delta)\right|-(p-1)\left|\operatorname{Re} H_{p}(u, \delta)\right|^{2} \\
& \leqq \frac{1}{4(p-1)}\left|\operatorname{Re} I_{p}(u, \delta)\right|^{2}
\end{aligned}
$$

and hence

$$
\left|\operatorname{Im} I_{p}(u, \delta)\right| \leqq(|p-2| / 2 \sqrt{p-1}) \operatorname{Re} I_{p}(u, \delta) .
$$

Going to the limit $\delta \downarrow 0$, we obtain (5).

\section{REMARKS}

1. First we note that $\tan ^{-1} x+\tan ^{-1}(1 / x)=\pi / 2 \quad(x>0)$. Consequently, we have

$$
\omega_{p}=\tan ^{-1}\left(\frac{|p-2|}{2 \sqrt{p-1}}\right)=\frac{\pi}{2}-\tan ^{-1}\left(\frac{2 \sqrt{p-1}}{|p-2|}\right) .
$$

Set $\varphi_{p}:=\frac{\pi}{2}-\omega_{p} \quad(1<p<\infty)$. Then $\varphi_{p}$ is found in Fattorini [1, Theorem 4.3 .1$, p. 188]; note that

$$
\frac{2 \sqrt{p-1}}{|p-2|}=\left[\left(\frac{p}{p-2}\right)^{2}-1\right]^{1 / 2} \quad(p \neq 2) .
$$

Fattorini considered a class of second order ordinary differential operators in $L^{p}(0, l)$, under various boundary conditions, as the infinitesimal generators of $\left(C_{0}\right)$-semigroups $\left\{S_{p}(\zeta)\right\}$ such that $S_{p}(\zeta)$ is analytic in the sector $|\arg \zeta|<\varphi_{p}$, and for $\omega>0, e^{-\omega \zeta} S_{p}(\zeta)$ is a contraction in a "smaller" sector. This is caused by the generality of the operators (see also Kato [5, Example V-3.34]).

2 . Set $p^{\prime}:=p(p-1)^{-1}$. Then it follows that

$$
\frac{\left|p^{\prime}-2\right|}{2 \sqrt{p^{\prime}-1}}=\frac{|p-2|}{2 \sqrt{p-1}} \quad(1<p<\infty)
$$

namely, the constant is the same in the adjoint space $L^{p^{\prime}}(\Omega)$.

3. We have started with the operator $A_{p}$ defined by (2) and (3). But the boundary condition was used just to get the equality (10). So we see that 
the sectorial estimate (5) is true for those operators satisfying the boundary conditions which lead to $(10)$.

4. The estimates (5) and (8) hold even if $\Omega=R^{m}$ and, for example, $A_{p}=-\Delta$ with $D\left(A_{p}\right)=W^{2, p}\left(R^{m}\right)$ and $\left\{T_{p}(t)\right\}$ is the semigroup generated by $-A_{p}=\Delta$; note that $C_{0}^{\infty}\left(R^{m}\right)$ is a core for $A_{p} \quad(1<p<\infty)$, and the (normalized) duality map is continuous (see Pascali-Sburlan [6, III- $\$ 2]$ ). (8) may be used to improve an estimate in Hempel-Voigt [4, Theorem 2.2(c)]. In fact, they used Stein's estimate (9) in the proof.

\section{ACKNOWLEDGMENT}

The author would like to thank the referee.

Added in proof. For the Laplacian, the constant $\tan \omega_{p}=(1 / 2)|p-2| / \sqrt{p-1}$ has already appeared in [10, p. 32] (see also [9] which was a preprint when the book [1] was being written). In this connection we note that the constant is best possible in the following sense: Suppose that there is a constant $C>0$ such that

$$
\left|\operatorname{Im}\left(-\Delta u,|u|^{p-2} u\right)\right| \leqq C \operatorname{Re}\left(-\Delta u,|u|^{p-2} u\right), \quad u \in W^{2, p}(\Omega) \cap W_{0}^{1, p}(\Omega) .
$$

Substituting a suitable function in this inequality, we can obtain $C \geqq \tan \omega_{p}$. This fact was first pointed out by Prof. S. Miyajima (Science Univ. of Tokyo) in the case of $\Omega=R^{2}$. The author expresses his hearty thanks to Prof. Miyajima.

\section{REFERENCES}

1. H. O. Fattorini, The Cauchy problem, Encyclopedia Math. Appl., vol. 18, Addison-Wesley, Reading, MA, 1983; Cambridge Univ. Press, New York, 1984.

2. J. A. Goldstein, Semigroups of linear operators and applications, Oxford Univ. Press, New York, 1985.

3. D. Gilbarg and N. S. Trudinger, Elliptic partial differential equations of second order, Grundlehren Math. Wiss., vol. 224, Springer-Verlag, Berlin and New York, 1977; 2nd ed., 1983.

4. R. Hempel and J. Voigt, On the $L_{p}$-spectrum of Schrödinger operators, J. Math. Anal. Appl. 121 (1987), 138-159.

5. T. Kato, Perturbation theory for linear operators, Grundlehren Math. Wiss., vol. 132, Springer-Verlag, Berlin and New York, 1966; 2nd ed., 1976.

6. D. Pascali and S. Sburlan, Nonlinear mappings of monotone type, Sijthoff \& Noordhoff International Publ., Alphen aan den Rijn, 1978.

7. A. Pazy, Semigroups of linear operators and applications to partial differential equations, Appl. Math. Sci., vol. 44, Springer-Verlag, New York, 1983.

8. E. M. Stein, Topics in harmonic analysis related to the Littlewood-Paley theory, Ann. of Math. Studies no. 63, Princeton Univ. Press, Princeton, NJ, 1970.

9. H. O. Fattorini, On the angle of dissipativity of ordinary and partial differential operators, Functional Analysis, Holomorphy and Approximation Theory. II (G. I. Zapata, ed. ), Math. Studies, vol. 86, North-Holland, Amsterdam and New York, 1984, pp. 85-111.

10. D. Henry, Geometric theory of semilinear parabolic equations, Lecture Notes in Math., vol. 840, Springer-Verlag, Berlin and New York, 1981.

Department of Mathematics, Science University of Tokyo, Wakamiya-Cho 26, SHINJUKU-KU, TOKYO 162, JAPAN 